\title{
Widerstand im Kollektiv
}

\author{
Bericht zur Tagung Jenseits der Person. \\ Die Subjektivierung kollektiver Subjekte an der \\ Universität Leipzig, 6.-8. April 2016
}

\section{Karsten Schubert*}

Die Tagung Jenseits der Person. Die Subjektivierung kollektiver Subjekte reagierte auf eine Lücke zeitgenössischer Subjektivierungstheorien. Subjektivierung wird mit und nach Foucault typischerweise als die Konstitution eines Individuums als ein Subjekt durch Macht verstanden. Subjektivierung bezieht sich also auf individuelle Subjekte. Was aber ist mit kollektiven Subjekten? Werden nicht auch sie subjektiviert, also als ein Subjekt durch Macht konstituiert? Kann der mittlerweile weit entwickelte Werkzeugkasten der Subjektivierung auch auf Kollektive angewendet werden? Um diese Dimension kollektiver Subjektivierung kreisten die Beiträge der zweieinhalbtätigen Tagung, die von Thomas Alkemeyer (Oldenburg), Ulrich Bröckling (Freiburg), Tobias Peter (Freiburg) und Martin Saar (Leipzig) vom 6. bis 8. April in Leipzig organisierte wurde.

Ulrich Bröckling setzte in seinem einleitenden Vortrag eine Differenz, die in vielen Diskussionen während der Tagung wieder aufgenommen wurde: Während Subjektivierung durch Kollektive in der individualistischen Konzeption verbleibe - ein Individuum wird durch die Macht eines Kollektivs subjektiviert und subjektiviert sich selbst sei unklar, was genau mit einer Subjektivierung von Kollektiven gemeint sein könne, durch die Kollektive erst konstituiert wür-

Karsten Schubert, M.A., Universität Duisburg-Essen Kontakt: karsten.schubert@uni-due.de den. Bröckling schloss seinen Vortrag mit dem Hinweis auf die widerständige und unterbrechende Funktion von Subjektivierung, die sich vor allem bei Rancière ausgearbeitet findet, und eröffnete damit eine zentrale Frage der Tagung: Was ist das Verhältnis von politischem Widerstand und der Konstitution von Kollektiven?

Im ersten Panel Theoretische Zugänge eröffneten Oliver Flügel-Martinsen und Franziska Martinsen die Konferenz, indem sie Subjektivierung als grundlegendes Problem der politischen Theorie beschrieben: die Spannung zwischen Unterwerfung und widerständiger Handlungsfähigkeit, die auch schon vor Foucault von Marx verhandelt wurde. Hannes Glück untersuchte anhand des Ausrufs „Wir sind das Volk“, der 1989 in Leipzig und heute von Pegida gerufen wird, politische Subjektivierungen in den Theorien Rancières und Laclaus. Das bei Pegida gemeinte Volk als ethnisch bestimmtes entspreche nicht Rancières normativem Politikbegriff, dessen Kriterium die unbedingte Gleichheit sei. Glück schlug vor, Laclaus in den Grundbegriffen normativ enthaltsamere Konzeption durch einen arbeitsteiligen Einsatz beider Theorien nutzbar zu machen. Wolfgang Fach brachte historische Gesellschaftstheoretiker und britische Politiker innen in einen Dialog und kontrastierte mit ihrer Hilfe verschiedene Modelle, das Verhältnis von Individuum und Gesellschaft zu denken. Gesellschaftliche Probleme könnten nur von Theorien in den Blick ge- 
nommen werden, die überhaupt einen Gesellschaftsbegriff haben, also holistisch und nicht atomistisch sind. Grundsatzfragen bestimmten die Diskussion im ersten Panel: Martin Saar vertrat die These, dass Rancières Gebrauch des Begriffs Subjektivierung als Emanzipation von Ausgeschlossenen völlig anders gelagert sei als der Foucault'sche, bei dem es um Subjektkonstitution als Unterwerfung und Entstehung von Handlungsfähigkeit geht. Es würde beiden Theorielinien nicht gerecht werden, sie einfach $\mathrm{zu}$ verschmelzen, wie Saar es im Beitrag von Flügel/FlügelMartinsen und in der Fragestellung der Tagung vermutete. Unterwerfung und kollektivpolitische Ermächtigung so in einem Begriff zusammenzubringen, sei ein begrifflicher Trick. Dass die beiden Perspektiven zusammengebracht werden sollten, war den anderen Diskussionteilnehmer_innen einleuchtender, schließlich wurde politische Handlungsfähigkeit gerade aus der Foucault'schen Linie heraus problematisiert - zu überprüfen sei allerdings, ob und wie genau Kollektive dabei helfen könnten, handlungsfähig zu werden.

Im Technologien betitelten zweiten Panel wurde das Problem der politischen Handlungsfähigkeit wieder aufgenommen durch eine Untersuchung der Behindertenbewegung von Lisa Pfahl und Boris Traue, wobei sie den Körper als Ansatzpunkt für Widerstand bestimmten. Kritisch diskutiert wurde diese Engführung der Subjektivierung mit Körperlichkeit und ob die Strategie der Behinderten tatsächlich ein Beispiel für die Subjektivierung kollektiver Subjekte sei. Thorsten Schlee analysierte den Begriff der Selbstorganisation kritisch. Er konstatierte eine normative Verbindung von Republikanismus und Informationstheorie, in der normative Ideale der Demokratie mit Effizienzerwartungen Hand in Hand gehen. In der Diskussion konnte durch eine Unterscheidung zwischen Selbstorganisation als reflexiver Organisation und als Emergenz neues Licht auf den immer noch vagen Begriff des Kollektivs geworfen werden: Wenn ein Kollektiv ein Ort für Emanzipation und Widerstand sein soll, dann kann es nicht als eine emergente Ordnung konzipiert werden, die ein Eigenleben gegenüber ihren Mitgliedern entwickelt, sondern muss durch sie steuerbar sein; kurz: demokratisch kann das Kollektiv nur als atomistisches, nicht als holistisches sein.

Politische Kollektivsubjekte sollten im dritten Panel genauer bestimmt werden. Jasmin Siri brachte zur Analyse von konservativ-rechten politischen Bewegungen zwei weitere Theorietraditionen zusammen, die Systemtheorie und die Psychoanalyse, mit denen sie nach der Wechselwirkung von Psyche und Sozialem fragte und die Emergenz eines politischen Selbst konzipierte. Felix Heidenreich trat mit der These an, dass Analogien und Metaphern neben Imperativen und Narrativen die dritte Form der Subjektivierung seien und kartographierte zum Beleg die „Baustelle“ als eine subjektivierende Metapher, die heute so dominant sei, dass man gar von einer „Ausweitung der Bauzone" sprechen könne. In der Diskussion herrschte Uneinigkeit über die Wirkmächtigkeit von Metaphern und die Möglichkeit, sie subversiv umzukehren. Für Martin Saar war nicht klar, ob der Beitrag sich auf den Hinweis beschränkte, dass nicht nur, wir Linken' Antiessentialisten seien, sondern auch ,die Macht' diese postfundamentalistische Lektion gelernt hätte und sie strategisch umsetze, wie dies die Gouvernementalitätsanalysen schon länger zeigen. Christian Helge Peters untersuchte die Konstitution von Bürgerwehren über den Affekt der Angst. In der Diskussion ging es um den Status von Affekten, die nicht grundsätzlich widerständig und auch nicht grundsätzlich konservativ seien. Im vierten Beitrag analysierte Helene Gerhards Patientenorganisationen und damit das erste widerständige und demokratische Kollektiv des Panels unter der Fragestellung, wie zunächst nichtpolitische Subjekte (durch Kollektive) politische werden. In der Diskussion wurde die Frage nach dem Status des Kollektivsubjekts wieder aufgenommen: Werden im Fall der Patientenorganisation nur Einzelsubjekte durch das Kollektiv subjektiviert, oder wird das Kollektiv selbst sub- 
jektiviert? Zwar wurde darüber keine Einigkeit erzielt, aber der Fall der Patientenorganisation wurde als Indiz dafür verstanden, dass zumindest ein gewisses Level an klassischer politischer Organisationsstruktur nötig ist, damit Kollektivsubjekte als politisch widerständig wirken können.

Bei der Frage nach dem sozialontologischen Status des Kollektivsubjekts gaben die dichten Beschreibungen der Mikrokollektive in Sport und Kunst in Panel vier Aufschluss. Am Beispiel des Volleyballspiels analysierte Matthias Michaeler komplexe Gruppenkonstitutionsprozesse durch die Schaffung einer über die Summe ihrer Mitglieder hinausgehenden Einheit des Sportteams und durch Training als intensivierte Praxis. Dafür, dass Kollektive emergent gegenüber ihren Mitgliedern sind, konnte Christian Müller durch seine Beschreibung des Zusammenspiels einer Jazzband weitere Indizien liefern. Er beschrieb die Interaktion der Band als einen temporären Subjektivierungsprozess. Die Emergenz des Ganzen funktioniert hier über die Zurücknahme der Handlungsabsichten des Einzelnen. In der Diskussion explizierte Felix Heidenreich die politische Relevanz eines in den Beiträgen deutlich gewordenen Verhältnisses: Für die Emergenz des Kollektivs als eigenständiger Akteur ist es notwendig, dass seine Mitglieder disziplinär subjektiviert sind (hartes Training, ständiges Üben) und dass sie eigene Handlungsmacht zurückstellen. Das Verhältnis von individueller Freiheit und kollektiver Wirkmächtigkeit ist deshalb spannungsreich. Das Verhältnis von Künstlern zu ihren Künstlerkollektiven untersuchten Severin Marguin und Cornelia Schendzielorz mit einem Methodenmix aus den Theorien Bourdieus, Foucaults und der Grounded Theory. In der Diskussion wurde die Frage aufgeworfen, wie die Emergenz des Kollektivs überhaupt festgestellt werden kann - durch eine Beschreibung der Emergenzerfahrung ihrer Mitglieder, wie bei den Künstlern, oder durch eine vom Erleben der Akteure unabhängige Sozialtheorie und Praxisanalyse, wie bei den Sportlern und Musikern.
Nach den Mikrokollektiven untersuchte das fünfte Panel Große Kollektive. Florian Heßdörfer beschrieb die Generation als ein kollektives Subjekt. Dafür wählte er einen pädagogischen Zugang und analysierte Kants Vorlesungen zur Pädagogik, die von Fortschrittsglauben geprägt seien. Heute hingegen ginge es der Pädagogik nicht mehr um die Gattungsanlage der Menschheit, sondern um die Ausbildung individueller Anlagen. Elena Dingersen sprach über die Stadt als Figur des Sozialen und analysierte dafür literarische Stadtcharakterisierungen der Bewohner_innen von Sankt Petersburg und Dresden. Imke Schmincke beschrieb die Frauenbewegung als ein Kollektivsubjekt mit Rückgriff auf die Methoden der Bewegungsforschung. Kollektive Identitäten bräuchten einen gemeinsamen kognitiven und narrativen Deutungsrahmen; den Deutungsrahmen der Frauenbewegung in der differenzfeministischen Phase untersuchte Schmincke anhand von programmatischen Texten von Alice Schwarzer und Verena Stefan. Diese Texte würden Frauen auffordern, ihre Körper und Sexualität zu entdecken und sich anzueignen, wobei das individuelle Schicksal der einzelnen Frauen auf das Kollektivschicksal aller Frauen zurückgeführt werde. Dies sei eine doppelte Subjektivierung, in der die Frauen erst durch den $\mathrm{Zu}$ sammenschluss ins Kollektiv zu individuellen autonomen Subjekten werden könnten. In der Diskussion wurde aus einer Butler'schen Perspektive gefragt, wie nötig eine Identität als ,Frau' sei, um frauenbewegt $\mathrm{zu}$ sein. Schmincke betonte eine historische Differenz in der Selbstbeschreibung von Frauen: In den 1980ern sei die Frauenbewegung als Kollektiv viel präsenter gewesen und die Kollektivität hätte eine wesentlich größere Bedeutung gehabt als heute, wo die individuelle Selbstverwirklichung für Frauen wichtiger sei.

Das letzte Panel war der Selbstreflexion gewidmet und beobachtete Kollektivsubjekte in Bildung und Wissenschaft. Um zu analysieren, wie Universitäten zu Subjekten werden, kombinierte Verena Eickhoff einen neoinstitutionalistischen und gouvernementalitätsanalytischen Ansatz. Den Diversity- 
Diskurs analysierte Eickhoff als eine Strategie von Hochschulen, um zu einer Akteurin zu werden bzw. den Akteurstatus zu sichern, beispielsweise indem sie herausragende Diversity-Programme entwickelten. Im letzten Beitrag der Tagung beschrieben Melanie Schmidt und Daniel Diegmann, wie in der Schule durch Zielvereinbarungen verschiedene Individuen zu einem Wir zusammengefasst werden, wodurch die Schule erst als kollektiv verfasster Akteur hervorgebracht werde.

In die Abschlussdiskussion führten Norbert Ricken und Nikolaus Buschmann durch einen rückblickenden Kommentar ein. All die auf der Tagung besprochenen Phänomene - von kollektiven Vollzügen (Volleyball, Jazz) über Netzwerke und Bewegungen (Frauen, Patienten, Bürgerwehren) bis zu Organisation (Parteien, Unis) - hätten gemeinsam, dass sie sich nicht individualtheoretisch beschreiben ließen. Diesen Kollektiven sei ein Subjektstatus zugeschrieben worden, weil sie handlungsfähig seien oder so adressiert, angerufen und aufgeladen würden. Offen blieb für Ricken und Buschmann allerdings, ob es tatsächlich Subjektivierung von (und nicht nur durch) Kollektive(n) gebe, mit anderen Worten, ob es sinnvoll sei, von Kollektiven als Handlungssubjekte zu sprechen. Diese emergente Ebene der Handlungsfähigkeit des Kollektivs sei bis zuletzt ungeklärt geblieben, obwohl die dichten Beschreibungen, beispielsweise der Jazz-Band, bei ihrem Ver- ständnis geholfen hätten. Um diesem Problem zu begegnen, schlug Ricken vor, die sozialphilosophische Diskussion über WirIntentionalität $\mathrm{zu}$ rezipieren und einzubeziehen. Unklar sei auch geblieben, wie die funktionalistisch-analytische Auffassung von Subjektivierung als Unterwerfung und Herstellung von Ordnung (Foucault) mit der normativen Auffassung von Subjektivierung als Kritik, Unterbrechung und Überschreitung der polizeilichen Ordnung (Rancière) zusammen gedacht werden könne.

In der Subjektivierungstheorie ist das Verhältnis von Unterwerfung und der Entstehung von widerständiger und freier Handlungsfähigkeit ein immer noch ungelöstes Grundproblem. Dabei wird oft implizit unterstellt, dass kollektive Zusammenschlüsse die Bedingung für die Entwicklung solcher Handlungsfähigkeit sind, ohne kollektive Subjektivierung dafür zu problematisieren. Dies endlich zu tun, dafür war die Tagung ein erster Schritt. Der sozialontologische Status von Kollektiven (atomistisch-reduzierbar/holistisch-emergent) und die Voraussetzungen dafür, dass Kollektive politisch widerständig sein können, blieben aber weitgehend ungeklärt. Nur wenn diese Fragen klarer beantwortet werden, könnte ein Konzept der Subjektivierung von Kollektiven auch zur Lösung des Problems der individuellen Handlungsfähigkeit in der Subjektivierungstheorie beitragen. 